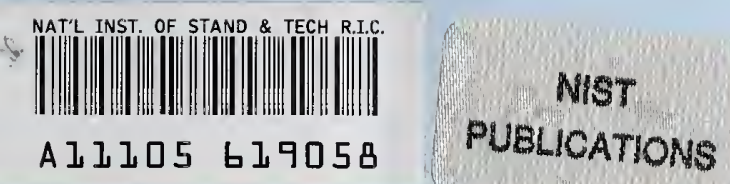

NISTIR 6358

\begin{abstract}
An Examination and Assessment of Available Incoherent Scattering S-Matrix Theory, also Compton Profile Information, and Their Impact on Photon Attenuation Coefficient Compilations
\end{abstract}

\section{J. H. Hubbell}

U.S. DEPARTMENT OF COMMERCE Technology Administration National Institute of Standards and Technology 100 Bureau Drive Stop 8463

Gaithersburg, MD 20899-8463 


$$
\text { - }
$$




\section{An Examination and Assessment of Available Incoherent Scattering S-Matrix Theory, also Compton Profile Information, and Their Impact on Photon Attenuation Coefficient Compilations}

\section{J. H. Hubbell}

U.S. DEPARTMENT OF COMMERCE

Technology Administration

National Institute of Standards and Technology

100 Bureau Drive Stop 8463

Gaithersburg, MD 20899-8463

July 1999

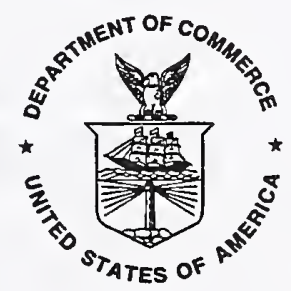

U.S. DEPARTMENT OF COMMERCE William M. Daley, Secretary

TECHNOLOGY ADMINISTRATION Gary R. Bachula, Acting Under Secretary for Technology

NATIONAL INSTITUTE OF STANDARDS AND TECHNOLOGY

Raymond G. Kammer, Director 
An Examination and Assessment of Available Incoherent Scattering S-Matrix Theory, also Compton Profile Information, and their Impact on Photon Attenuation Coefficient Compilations*

\author{
J. H. Hubbell ${ }^{+\#}$
}

11830 Rocking Horse Road, Rockville, MD 20852

\begin{abstract}
The Klein-Nishina formulae for the differential and integral cross sections for Compton (or incoherent) scattering of $x$-ray photons from atomic electrons assume that the electrons are free (unbound) and at rest, which is a good approximation for photons of the order of $1 \mathrm{MeV}$ or higher, particularly for low- $Z$ target materials. The probability for dislodging bound electrons can be taken into account at least approximately by including an incoherent scattering function $S(x, Z)$ in the differential Klein-Nishina formula. Currently available systematic theoretical tabulations of $S(x, Z)$, where $\mathrm{x}$ is a momentum transfer variable dependent on the photon deflection angle and the photon incident energy do not use S-matrix theory with its claimed higher accuracy. The currently most widely-used S(x,Z) tables are non-relativistic. Recently (1998) Kahane has used the RibberforsBerggren relativistic treatment to compute systematic tables of $S(x, Z)$. These new values of $S(x, Z)$ have not yet been exploited for computing relativistic bound-electron incoherent scattering integrated cross sections $\sigma_{\text {incoh }}$, nor for triplet (pair production in the field of the atomic electrons) cross sections $\kappa_{e}$ which also require $S(x, Z)$ for their computation. The motion of the atomic electrons around the atomic nucleus gives rise to a Doppler broadening of the apparent energy of the incident photon, resulting in a corresponding broadening of the Compton "modified line" for a given deflection angle of the outgoing scattered photon. The shape of this broadened line is called the "Compton profile." One task of this report is to examine the usefulness and advantages, if any, of S-matrix theory in providing significantly more accurate values of photon interaction cross sections and attenuation coefficients. The other task of this report is to examine the available Compton profile literature and to explore what, if any, effect our knowledge of this line-broadening has on theoretical computations of photon incoherent scattering cross sections and total mass attenuation coefficients.
\end{abstract}

Key Words: Compton profile, cross section, gamma-ray, mass attenuation coefficient, photon, Smatrix theory, $\mathrm{x}$-ray

"Work supported by the Office of Standard Reference Data of the National Institute of Standards and Technology.

+Work carried out for the National Institute of Standards and Technology under contract 43NANB809980.

"To whom all correspondence should be addressed at: National Institute of Standards and Technology, Mail Stop 8463, Gaithersburg, MD 20899-8463 


\section{Introduction.}

\section{S-Matrix Incoherent Scattering Theory:}

The availability of S-matrix theory for computing photon-atom interaction cross sections was briefly touched on in a previous report in this series (Hubbell 1997). This report will now endeavor to estimate the magnitude of the anticipated impact and possible adjustments in values of the mass attenuation coefficients $\mu / \rho$ from those in the above compilations, in changing the theoretical bases for systematically computing the incoherent scattering cross section $\sigma_{\text {incoh }}$ from those of Hubbell et al (1975) based on the work of Brown (1970a,b, 1972, 1974), of Cromer and Mann (1967a,b) and of Cromer $(1969,1971)$ to the S-matrix approach. The S-matrix approach is outlined, for example, in a series of papers by Bergstrom et al. (1992, 1993a,b, 1995, 1997) and by Pratt, Kissel and Bergstrom (1994). In the survey in Section 2, and in the conclusions in Section 4, the feasibility and implications of S-matrix incoherent-scattering theory for computations of the Compton and triplet (Hubbell et al 1980) contributions to the energy-absorption coefficient $\mu_{e n} / \rho$ (Seltzer 1993) is also explored.

\section{Available Data on Compton Profiles:}

Since the impact of Compton profile data on the computation of $x$-ray cross section and attenuation coefficients seems not to have been explored previously in the literature, the major part of this report, Section 3, will focus on this second topic.

In the early experimental results by Gray $(1920)$, and by Compton $(1922,1923)$ on the $x$-ray scattering effect bearing his name, and in the theoretical work by Debye (1923), by Dirac (1926), by Klein and Nishina (1929), by Compton (1935), and later by Platzman and Tzoar (1977), little attention was given to the apparent broadening in energy of the deflected photon, due to the poor resolution of the early spectrometers. Some early theory was provided by Jauncey (1925a,b, 1934), after which some definitive measurements of this broadening, or "Compton profile", were made by Du Mond $(1929,1930,1933)$ and Du Mond and Kirkpatrick $(1931,1937)$ using an X-ray tube and spectrometer arrangement especially designed for Compton scattering. An excellent recent review of subsequent measurements and theory of the Compton profile has been given by Cooper (1997).

As discussed by Duncanson and Coulson (1945), who obtained closed-form analytical expressions for the Compton line-shapes for isolated (unbound) atoms He through $\mathrm{Ne}$, if a monoenergetic photon beam falls on an atom in which all the electrons are at rest, the scattered photons at any given deflection angle should have the single energy predicted by the Compton $(1922,1923)$ and Debye (1923) formula. However, if the electrons are in motion, as we know to be the case, there is a Doppler effect related to the projected velocities of the electrons. This causes the modified (Compton) line to spread out and become a band, whose profile can then be used to determine the electron velocity distribution. This Doppler-based description (Du Mond 1933) gives effectively the same results as the complete wave-mechanical theory of Waller (1927) and Wentzel $(1927 a, b)$.

The newly available high intensity and higher-energy synchrotron radiation sources, heading toward the 50 to $500 \mathrm{keV}$ range, according to Cooper (1997), make possible the exploitation of Compton profile measurements to examine the wave-mechanical structures of the scatterers. For example, Compton profiles can be used to check band theoretical models of solids, to identify electron 
correlation effects, and recently have been used to extract Fermi surface topography in Li metal (Schülke et al 1996).

Clearly, high-resolution Compton profile measurements are a useful tool for a variety of atomic structure and solid state investigations. However, the impact of our knowledge of these profiles on general-use systematic compilations of X-ray attenuation coefficients, $\mu / \rho$, such as, for example, those of Berger and Hubbell (1987, 1998/1999), Creagh and Hubbell (1992), Hubbell and Seltzer (1995) .and of Cullen, Hubbell and Kissel (1997), also on systematic calculations and compilations of the incoherent scattering cross section $\sigma_{\text {incoh }}$ such as in Hubbell et al (1975), has not yet been specifically treated in the literature, to this author's knowledge. It is the aim of this report, in the survey in Section 3, and in the conclusions in Section 4, to provide a basis for such a treatment.

\section{Survey of S-Matrix Theory.}

A concise definition of the S-matrix (scattering matrix, also sometimes called the collision matrix) is given in The International Dictionary of Physics and Electronics (Michels 1961): "Consider a quantum mechanical system composed of two parts, such as (1) an atom or nucleus, and (2) the electromagnetic radiation field, or such as (1) an incoming proton and (2) a nucleus. It is possible to express the initial state of the system as a linear combination of eigenfunctions. After a photon of the electromagnetic field has been scattered from the atom or nucleus, or, for example in the case of particle-radiation interactions, after a proton has been scattered from a nucleus, the final state of the system can again be expressed as a different linear combination of the same set of eigenfunctions as that used in the initial description. If the wave functions for the initial and final states are $\psi(t=-\infty)$ and $\psi(\mathrm{t}=+\infty)$ respectively, and are given by the expressions

$$
\begin{aligned}
& \psi(\mathrm{t}=-\infty)=\Sigma_{\mathrm{a}} \mathrm{a}_{\mathrm{m}} \psi_{\mathrm{n}}, \text { and } \\
& \psi(\mathrm{t}=+\infty)=\Sigma_{\mathrm{m}} \mathrm{b}_{\mathrm{m}} \psi_{\mathrm{m}},
\end{aligned}
$$

in terms of the system's eigenfunctions $\psi_{n}$, then the matrix having elements $S_{m n}$, such that

$$
b_{m}=\Sigma_{n} S_{m n} a_{n}
$$

is called the S-matrix."

According to Newton (1982), the S-matrix was first independently introduced in quantum scattering theory by Wheeler (1937) and by Heisenberg (1943 a,b), and is sometimes referred to as the S matrix of Heisenberg (Goldberger and Watson 1964). Treatments of the S-matrix and its applications appear in many standard textbooks, for example Blatt and Weisskopf (1952), Ballentine (1998) and in particular Rodberg and Thaler (1967) which devotes an entire chapter to the S-matrix and the Kmatrix, the latter of which conveys the same information as the S-matrix but has different properties.

The first significant application of the S-matrix formalism to Compton scattering appears to be that by Whittingham $(1971,1981)$ in which, according to Suric et al. (1991), the external field was assumed to be point Coulombic (unscreened), and a limited number of partial waves and multipoles were included in the calculations. Whittingham's results, according to Suric et al. (1991), were significantly larger than the predictions of more approximate methods, for the cases that were 
considered, and the computational codes could not be verified. Another early application of S-matrix formalism to Compton scattering was that by Wittwer (1972).

As yet, there appear to be no systematic (all-Z, all-energies) computations and tabulations of Compton cross sections, where $Z$ is the atomic number, utilizing the $S$-matrix formalism, in a form amenable to incorporation in systematic (all-Z, extended photon energy range) photon cross section and attenuation coefficient data bases. The existing available theoretical tools for improved Compton scattering computations, including "exact" second-order relativistic S-matrix prescriptions, have recently been reviewed by Bergstrom and Pratt (1997). This review outlines the recent Compton theory progress to date, primarily from the efforts of a series of collaborations between researchers at the University of Pittsburgh, the Lawrence Livermore National Laboratory, and the Ruder Bošković Institute in Zagreb, Croatia (Surić et al. 1991, Surić 1992, Bergstrom et al. 1992, Bergstrom 1993a, Bergstrom et al. 1993b, Pratt et al. 1994, Bergstrom 1995 and Surić et al. 1996). In this series of collaborations, an exact second-order S-matrix code has been developed for the relativistic numerical calculation of cross sections for Compton scattering of photons by bound electrons within the independent particle approximation (IPA). In the sample cases studied, comparison with measurements is found to be better than with impulse approximation calculations.

The recent review by Kane (1997) of experimental studies of inelastic (Compton) scattering of x-ray and $\gamma$-ray scattering by atoms includes some comparisons with $S$-matrix computations as well as with the non-relativistic Brown/Cromer incoherent scattering factor $S(x, Z)$ systematic compilation of Hubbell et al. (1975). Because the quantity $S(x, Z)$ was introduced in the $x$-ray crystallography literature, the momentum transfer parameter $\mathrm{x}$ is customarily expressed in terms of the photon wavelength $\lambda$ in ångstroms $(\AA)$, where $1 \dot{A}=10^{-10} \mathrm{~m}$, rather than in terms of the photon energy $E$ (e.g., in $\mathrm{keV}$, where $1 \dot{\mathrm{A}}=12.3984244 / \mathrm{E}(\mathrm{keV})$, Cohen and Taylor 1986,1997$)$ for tabulation purposes. Thus in the definition: $\mathrm{x}=\sin (\theta / 2) / \lambda(\dot{\mathrm{A}})$, where $\theta$ is the scattered-photon deflection angle, $\mathrm{x}$ has the units $\AA^{-1}$. In the example in Kane (1997) for $\mathrm{Sn}(Z=50)$, the measurement error bars for a Compton profile (279.2 $\mathrm{keV}, \theta=115^{\circ}$, where $\theta$ is the scattered-photon deflection angle) are too large to draw firm conclusions toward preferring the $S$-matrix results over the non-relativistic widelyused incoherent scattering function values. The comparisons by Namito et al. (1995) of recent measurements of $S(x, Z)$ with the Hubbell et al. (1975) compilation suggest that the latter compilation, in addition to its convenience, is still useful for most practical applications.

\section{Survey of Selected Compton Profile Measurements and Theoretical Treatments.}

The question has been raised, of whether incorporation of the effect of Doppler broadening on the spectrum of the Compton-scattered photon should have any significant effect on the calculation of the cross section for this process, for providing more-accurate general-purpose systematic tabulations of the mass attenuation coefficient $\mu / \rho$ and related quantities such as the mass energy-absorption coefficient $\mu_{\mathrm{en}} / \rho$. If one is interested in the spectral distribution of the Compton scattered photons, and not just the integrated cross section, Namito et al. (1994) have made it dramatically clear, by computing the scattered spectrum with and without including Doppler broadening in the EGS4 code (Nelson et al. 1985), that such broadening must be included in order to replicate the corresponding measurements by Namito et al. (1994) using $40 \mathrm{keV}$ polarized photons from a $2.5 \mathrm{GeV}$ synchrotron light source (KEK-PF).

This section presents a more-or-less chronological survey and account of selected experimental and theoretical studies of the Compton profile, or Doppler spectral broadening of what would be a 
monoenergetic scattered photon, for a monoenergetic source and a particular photon deflection angle $\theta$. Whether or not any conclusions can be drawn from this account, at the very least this section (and the references cited) provides a guide to on-file documents on this topic that are held in the NIST Photon and Charged Particle Data Center reference collection.

The first major theoretical treatment of the energy-broadening of the Compton scattered line appears to be that of Jauncey $(1925 a, b, 1934)$ who considered the kinetic energy, and hence its component distribution of velocities, of the electron in its Bohr orbit. The next step was the work by Waller (1927) who used the Kramers-Heisenberg dispersion formula to show that one should see a slightly broadened Compton line. Throughout the 1930s, extensive experimental and theoretical studies of the Compton line structure were carried out by Du Mond and Kirkpatrick (Du Mond 1929, 1930, Du Mond and Kirkpatrick 1931, 1937, Kirkpatrick and Du Mond 1938); much of this work was organized and presented in an extensive and definitive Reviews of Modern Physics article (Du Mond 1933). As with Jauncey's earlier work, Du Mond's careful measurements could to a good approximation be interpreted in terms of Doppler broadening due to the plus and minus velocity projections of the orbiting electrons.

During this same period, Kappeler (1936) made Compton profile measurements on gas targets (Ne, $\mathrm{O}_{2}$ and $\mathrm{N}_{2}$ ) and on solids ( $\mathrm{Li}, \mathrm{C}$ and $\mathrm{NaF}$ ), and Burkhardt made measurements on Ne. Schnaidt (1934) and Franz (1937) added refinements to the theoretical understanding of the process. Hicks (1937) used self-consistent-field variation functions which take into consideration the instantaneous interaction of the electrons, momentum distributions and intensity distributions to compute Compton lines for helium and molecular hydrogen. Thes computations neglected the small relativity and binding corrections.

Moving into the World War II period, Duncanson and Coulson (1945) derived closed analytical expressions for the shapes of the Compton lines scattered from the elements $\mathrm{H}$ to $\mathrm{Ne}$, in the form of free atoms. From their analysis of the then-available body of measurements, they concluded that by forming a diatomic molecule, the width of the Compton profile is increased by about $15 \%$ to $25 \%$.

Brini et al. (1960) measured the differential Compton cross section for $662 \mathrm{keV}$ photons on the Kshell electrons of $\mathrm{Pb}$, using the coincidence technique with the $\mathrm{K}$ x rays produced in the process, and with care to use $\mathrm{Cu}$ shielding to avoid detecting $\mathrm{K}$ x rays from the $\mathrm{Pb}$ collimation system. In this same period, Kilby experimentally investigated the electron momentum distributions in $\mathrm{H}_{2}$ and $\mathrm{He}$ (Kilby 1961) and in metallic Li (Kilby 1963), and concluded that there were some serious discrepancies between theory and experiment with respect to the mean kinetic energy of the conduction electrons, at least in Li. Motz and Missoni (1961) and Di Lazzaro and Missoni (1966) also measured Compton spectra from scattering by K-shell electrons of $\mathrm{Sn}, \mathrm{Au}$ and $\mathrm{U}$, and Varma and Eswaran (1962) measured Compton spectra from the $\mathrm{K}$-shell electrons of $\mathrm{Pb}$. All measurements were made with $662 \mathrm{keV}$ photons.

Kilby (1965) derived expressions for the Compton profile intensity based on the wave-mechanical scattering theory of Waller and Hartree (1929) assuming: (a) a one-electron approximation is valid, (b) electronic binding energies are small compared with the energy transferred from the photon, and (c) the electron wave functions for the relevant continuum states are approximated by plane waves. He concluded from this study that the shape of the Compton profile near its peak is particularly sensitive to the description of the more loosely bound electrons, and that hence the Compton profile measurements can yield information about the valence electrons of the heavier elements. Veigele et al. $(1966,1969)$ used a relativistically covariant formalism to derive a generalized Compton equation; 
the derived Compton profile showed a defect (a reduced intensity) in the broadened line toward a wavelength shorter than that predicted by the Compton equation.

Weiss and Phillips and collaborators (Weiss et al. 1968a, Weiss and Phillips 1968b, Phillips and Weiss 1968, Weiss 1972, 1973a,b, 1975, Phillips and Weiss 1972a,b, Phillips 1973, Weiss et al. 1977) exploited Compton profiles for examining valence electrons in solids, both experimentally and theoretically, and published extensive tables of Compton line shapes and electron momentum distributions for atoms and ions as heavy as $\mathrm{Ge}(Z=32)$. Schülke et al. (1969) measured directional Compton profiles for $\mathrm{Be}$, and found evidence for Coulomb interactions between electrons that were consistent with the RPA (random phase approximation) calculations of Ohmura and Matsudaira (1964) for photon inelastic scattering by an electron in a degenerate electron gas. In turn, Ohmura and Matsudaira (1964) noted a discrepancy between the Kappeler (1936) Li Compton profile measured width and the Wigner-Seitz wave function calculation by March (1954) and Donovan and March (1956) which could not be explained by Coulomb correlation. More recently, Schülke et al. (1996) used synchrotron radiation from the DORIS storage ring, monochromatized to $31 \mathrm{keV}$, to measure directional Compton profiles from $\mathrm{Li}$ metal. They found discrepancies from the recent densityfunctional calculations by Sakurai et al. (1995) that they attributed to self-energy effects connected with the excitation of so-called plasmaron modes.

Another extensive body of original and review work on Compton profiles is that by Cooper and collaborators (Cooper et al. 1970, 1974, 1976, Stuewer and Cooper 1977, Cooper 1985, Cardwell and Cooper 1986, Cooper 1997). In one of these works, Cooper (1997) points out that what has been called in common parlance "magnetic Compton scattering" should now be called "spin-dependent Compton scattering." During this same period, Eisenberger and collaborators (Eisenberger 1970, 1972a, Eisenberger et al. 1972b,c,d, Reed and Eiesenberger 1972, 1974) also have provided extensive Compton profile measurements and comparisons with theory. Their observations of highmomentum anisotropy in both $\mathrm{Cu}$ and $\mathrm{Ni}$ suggested d-electron bonding in the systems studied. Manninen and collaborators (Paakkari et al. 1972, 1973a,b, Manninen 1974, Paatero et al. 1974, Manninen 1978) developed an annular collimation arrangement with which they made Compton profile measurements with $\mathrm{Al}, \mathrm{V}$ and $\mathrm{Fe}$ targets. They found considerable deviations in their results from predictions of the free-atom, free-electron model at low momentum transfers. However, there was reasonable agreement for high momentum transfers.

Epstein (1973) measured Compton profiles in benzene and several other hydrocarbons, and Tanner and Epstein (1974) continued these studies with Compton profiles from a water target. From a comparison of their measurements with "near Hartree-Fock" (NHF) and "minimal basis set" (MBS) calculations, they concluded that neither energy minimization nor fulfillment of the virial theorem is in itself sufficient to guarantee a reliable momentum distribution.

Ribberfors (1975a) developed expressions for Compton profiles in the case of isotropic momentum distributions, and generalized these results (Ribberfors 1975b) to include the effects of both anisotropy and polarization. In a further work, Ribberfors and Berggren (1982) showed that incoherent scattering functions $S(x, Z)$, defined as an integral over Compton profiles, could easily ("on a pocket calculator") be computed by means of a linear approximation. However, their results for low Z materials were barely distinguishable from the non-relativistic $S(x, Z)$ compilation of Hubbell et al. (1975), both being well within the claimed uncertainties of available measurements, and for a high $\mathrm{Z}$ material (e.g., Pb) their results were completely indistinguishable. On the other hand, in a recent personal communication, Ribberfors (1999) cautioned that for low $\mathrm{Z}$ and low $\mathrm{x}$ there could be a substantial difference. For example, Ribberfors and Berggren (1982), found for $\mathrm{Al}$ and $\mathrm{x}<0.2 \AA^{-1}$ 
that the integrated Compton profile $S(x, Z)$ results were as much as $40 \%$ less than the Waller-Hartree non-relativistic S(x,Z) Cromer and Mann (1967b) and Cromer (1969) values incorporated into the Hubbell et al. (1975) compilation.

Williams et al. (1976), acting in behalf of the International Union of Crystallography Commission on Spin, Charge and Momentum Density, endeavored to standardize Compton profile measurements, taking water as their anchor material for this project. Five different laboratories participated, providing 13 independent Compton profile measurements on water. Inconsistent normalization of the various measured profiles was found to be the major source of error. After renormalization of the profiles, the results were found to be consistent to within $\pm 2 \%$ of the peak height of the profiles. It was concluded that reproducibility of $\pm 1 \%$ of the peak height of the profile is quite feasible, and that in a differential experiment $\pm 0.5 \%$ or even better is possible. The techniques are extensively reviewed in Williams (1977b), with further details in the chapters in Williams (1977a) by Halonen et al. (1977), Mendelsohn and Smith (1977) and by Berggren et al. (1977).

Owen $(1977,1978)$ theoretically examined both binding effects and "off-mass-shell" effects on the Compton profile using Dirac wave functions. From his calculations, he concluded that ignoring these effects for photons scattered in the forward directions from electrons in high-Z atoms distorts and extends the low-energy part of the profile. Further, he observed that these uncorrected profiles have secondary maxima which contribute a large spurious amount to the calculated cross section.

Pattison and Schneider (1979) made a test of the relativistic 1s wave functions in $\mathrm{Au}$ and $\mathrm{Pb}$ by measuring the Compton profiles in these elements using $412 \mathrm{keV}$ gamma radiation. Their observed energy dependence of the Compton scattering cross section was in good agreement with the expression given by Ribberfors (1975a), and they found good agreement between their measurements and the relativistic Hartree-Fock Compton profiles calculated by Biggs et al. (1975). In an extension of this work, Weyrich et al. (1979) examined the Compton profiles of atoms and molecules using Fourier analysis. In particular, they investigated the possibility of extracting accurate bond lengths, with the fluorine molecule $\mathrm{F}_{2}$ as an example.

Carlsson et al. (1982) have explored the inclusion of Compton profile effects on scattering cross section calculations for applications in diagnostic medical radiology. They provide a useful assortment of formulas and procedures for this purpose.

Schumacher's group (Reineking et al. 1983, Wenskus et al. 1985) calculated double differential bound Compton scattering cross sections in the relativistic impulse aproximation using DFHS (DiracHartree-Fock-Slater) wave functions. They found good agreement with their measurements (Schumacher 1971, Rullhusen and Schumacher 1976, Reineking et al. 1983) of Compton scattering of 279 and $662 \mathrm{keV}$ photons by $\mathrm{Cu}, \mathrm{Sn}$ and $\mathrm{Pb}$ targets at the peak of the profiles. However, on the high-energy sides of their measured profiles they noted discrepancies which were only partly explained by second-order S-matrix calculations. Kane et al. (1987) included Compton scattering measurements of $88.03 \mathrm{keV}$ gamma rays on a Bi target in their work which also included cross sections for elastic scattering and atomic photoeffect. They obtained an incoherent scattering value $S(x, Z)$ at $x=6.3 \AA^{-1}$ of $68.6 \pm 4.7$ to compare with the non-relativistic value of 77 in the Hubbell et al. (1975) compilation. This work is included in the extensive review by Kane (1992) of inelastic scattering of photons in the $\mathrm{X}$-ray and gamma-ray energy region.

Most recently, Deb et al. (1999) have measured directional Compton profiles for indium phosphide (InP) using gamma rays from ${ }^{241} \mathrm{Am}$ and compared their measured results with their calculated results 
which were based on the linear combination of Gaussian orbitals (LCGO) method. They found good agreement and that the anisotropy dependences have an intimate connection with the bonding properties of the semiconductor. This work was a continuation of the measurements by Bandyopadhyay et al. (1996) of Compton profiles of vanadium carbide and theoretical interpretation by Deb and Chatterjee (1996a) and measurements by Bandyopadhyay et al. (1994) of profiles of cuprous oxide and theoretical interpretation by Deb and Chatterjee (1996b) who used the LCGO model method. Further on work Compton profile measurements and theory by this group, including related properties such as density of states (DOS) and charge analysis, with emphasis on chemical bonding, is in progress.

\section{Conclusions and Recommendations.}

\section{S-Matrix Incoherent Scattering Theory:}

From the papers examined and described in Section 2, one can conclude that for Compton scattering of photons from electrons bound in atoms, differential and integrated cross sections for this process calculated using the S-matrix formalism should give values in closer agreement with measured values. However, there is a problem in presentation of the results for practical application, such as in EGS4 codes for transport calculations, and in other applications, since the data array for the equivalent of the now two-dimensional incoherent scattering function $S(x, Z)$ would become a three-dimensional function $S(E, \theta, Z)$ in which $E$ is the photon incident energy and $\theta$ is the scattered-photon deflection angle. With modern computers, such higher dimensional arrays are perhaps feasible.

However, as noted from the Namito et al. (1995) comparisons of incoherent scattering function measurements with theory, the non-relativistic but convenient two-dimensional $S(x, Z)$ values compiled by Hubbell et al. (1975) by combining and extrapolating the works of Brown (1970a,b, 1972, 1974), of Cromer and Mann $(1967 a, b)$ and of Cromer $(1969,1971)$ appear to be indistinguishable from the $S$-matrix formalism results, within the error bars of the available experiments, particularly for high- $Z$ elements. Ribberfors (1999) points out the significant differences between non-relativistic and relativistic impulse-approximation theoretical treatments for $\mathrm{Al}$ below $\mathrm{x}=0.2 \dot{\mathrm{A}}^{-1}$ (see. e.g., Figure 2 in Ribberfors and Berggren (1982)).

Recently, Kahane (1998) has used the Ribberfors-Bergren (1982) treatment to compute and tabulate relativistic Dirac-Hartree-Fock photon incoherent scattering functions $S(x, Z) / Z$ for all elements $1 \leq$ $\mathrm{Z} \leq 110$ for a range of values of the momentum transfer parameter $0.005 \dot{A}^{-1} \leq \mathrm{x} \leq 1000 \AA^{-1}$. The only measured $S(x, Z)$ values in this region, with which to compare and assess non-relativistic vs. relativistic treatments, are those of Laval (1942) [in France, at the height of the 1940-1944 World War II Nazi occupation] for which no error estimates are provided; also, little information is given on the experimental details. However, in the future, it is anticipated that relativistic $S(x, Z)$ values such as those by Kahane (1998) will replace the Hubbell et al. (1975) non-relativistic $S(x, Z)$ values, in both incoherent (Compton) scattering and triplet production cross section computations for $\mu / \rho$ compilation purposes.

\section{Measured and Theoretical Compton Profile Data:}

From the above survey in Section 3 of measured and theoretical Compton profile data, by its very nature of being sensitive to chemical bonding by valence electrons, it would be very difficult to systematically compile "engineering type" tables, such as is now done for the mass attenuation 
coefficients $\mu / \rho$ and incoherent scattering functions $S(x, Z)$, for scattering and attenuation data which depends on the bonding-sensitive Compton profiles. However, as dramatically illustrated by Namito et al. (1994), Doppler broadening should and can be incorporated into the EGS4 (Nelson et al. 1985) and other Monte Carlo photon-electron radiation transport codes, as shown by their comparisons of calculated and measured spectra for $\mathrm{C}$ and in $\mathrm{Cu}$.

\section{- Acknowledgements:}

The author thanks Prabhakar Kane, Roland Ribberfors, Richard Pratt and Paul Bergstrom for some suggestions and references. 


\section{References:}

Ballentine, L.E. (1998) Quantum Mechanics. A Modern Development (World Scientific, Singapore). p. 454.

Bandyopadhyay, S., Chatterjee, A.K., Saha, S.K. and Chatterjee, A. (1994) Compton profile of cuprous oxide. Rad. Phys. Chem. 44, 517-520.

Bandyopadhyay, S., Saha, S.K. and Chatterjee, A.K. (1996) Compton profile of vanadium carbide. Rad. Phys. Chem. 47, 807-810.

Berger, M.J. and Hubbell, J.H. (1987) XCOM: Photon cross sections on a personal computer. National Bureau of Standards (now National Institute of Standards and Technology) report NBSIR 87-3597.

Berger, M.J. and Hubbell, J.H. (1998/1999) Photon attenuation coefficients. in CRC Handbook of Chemistry and Physics, 79th Ed. (Boca Raton: CRC Press) pp. 10-207 to 10-211.

Berggren, K.-F., Manninen, S., Paakkari, T., Aikala, O. and Mansikka, K. (1977) Solids. Ch. 6 in B. Williams (ed.) Compton Scattering (McGraw-Hill, New York) pp. 139-208.

Bergstrom, P.M., Surić, T., Pisk, K. and Pratt, R.H. (1992) Some preliminary calculations of whole atom Compton scattering of unpolarized photons. Nucl. Instr. Meth. B 71, 1-6.

Bergstrom, P.M. (1993a) Inelastic scattering from bound electrons at synchrotron radiation energies. Nucl. Instr. Meth. B 79, 240-243.

Bergstrom, P.M., Surić, T., Pisk, K. and Pratt, R.H. (1993b) Compton scattering from bound electrons: Full relativistic independent-particle-approximation calculations. Phys. Rev. A 48, 1134-1162.

Bergstrom, P.M. (1995) Single and double ionization of atoms by Compton scattering. Nucl. Instr. Meth. B 99, 148-151.

Bergstrom, P.M. and Pratt, R.H. (1997) An overview of the theories used in Compton scattering calculations. Radiat. Phys. Chem. 50, 3-29.

Biggs, F., Mendelsohn, L.B. and Mann, J.B. (1975) Hartree-Fock Compton profiles for the elements. At. Data Nucl. Data Tables 16, 201-309.

Blatt, J.M. and Weisskopf, V.F. (1952) Chapter 10, Formal Theory of Nuclear Reactions, in Theoretical Nuclear Physics (Wiley, New York) pp.517-564,

Brini, D., Fuschini, E., Grimellini, N.T. and Murty, D.S.R. (1960) Compton effect on the bound electrons. Nuovo Cim. 16, 727-736.

Brown, R.T. (1970a) Coherent and incoherent x-ray scattering by bound electrons. I. Helium isoelectronic sequence. Phys. Rev. A 1, 1342-1347. 
Brown, R.T. (1970b) Coherent and incoherent $x$-ray scattering by bound electrons. II. Three- and four-electron atoms. Phys. Rev. A 2, 614-620

Brown, R.T. (1972) Incoherent-scattering function for atomic carbon. Phys. Rev. A 5, 2141-2144.

Brown, R.T. (1974) Coherent and incoherent x-ray scattering by bound electrons. III. Five-electron atoms. Phys. Rev. A 10, 438-439.

Burkhardt, G. (1936) Über die Form der Comptonlinie. Ann. Physik 26, 567-584,

Cardwell, D.A. and Cooper, M.J. (1986) Directional Compton profile measurements of aluminium with 412 and $60 \mathrm{keV}$ radiation. Phil. Mag. 54, 37-49.

Carlsson, G.A., Carlsson, C.A., Berggren, K.-F. and Ribberfors, R. (1982) Calculation of scattering cross sections for increased accuracy in diagnostic radiology. I. Energy broadening of Compton-scattered photons. Med. Phys. 9, 868-879.

Cohen, E.R and Taylor, B.N. (1986) The 1986 adjustment of the fundamental physical constants. CODATA Bull. 63 (Pergamon, Elmsford, N.Y.); [same title] Rev. Mod Phys. 57, 1121-1148 (1987); The 1986 CODATA recommended values of the fundamental physical constants. J. Res. Nat. Bur. Standards 92, 85-95 (1987); [same title] J. Phys. Chem. Ref. Data 17, 17951803 (1988).

Cohen, E.R. and Taylor, B,N. (1997) The fundamental physical constants [1986 values unchanged]. Physics Today (November 1997), BG9-BG16.

Compton, A.H. (1922) Secondary radiations produced by x-rays, and some of their applications to physical problems. Bull. Nat. Res. Council (U.S.) 4 (20), 1-56.

Compton, A.H. (1923) A Quantum theory of the scattering of x-rays by light elements. Phys. Rev. $21,483-502$.

Compton, A.H. and Allison, S.K. (1935) Scattering from bound electrons, Ch. III, Sec. 19 in X-Rays in Theory and Experiment. 2nd Ed. (Van Nostrand, New York), pp. 238-255. See also Appendix IV "Atomic structure or form factors", Table II "Incoherent scattering function, $\Sigma f^{2 ",}$ pp.780-782.

Cooper, M.J., Williams, B.G., Borland, R.E. and Cooper, J.R.A. (1970) Compton scattering and electron momenta in lithium. Phil. Mag. 22, 441-447.

Cooper, M.J., Pattison, P., Williams, B. and Pandey, K.C. (1974) The Compton profile of aluminium. Phil. Mag. 29, 1237-1243.

Cooper, M.J., Pattison, P. and Schneider, J.R. (1976) Compton profile measurements with $412 \mathrm{kev}$ $\gamma$-radiation. Phil. Mag. 34, 243-257.

Cooper, M.J. (1985) Compton scattering and electron momentum determination. Rep. Prog. Phys. $48,415-481$. 
Cooper, M.J. (1997) Compton scattering and the study of electron momentum density distributions. Radiat. Phys. Chem. 50, 63-76.

Creagh, D.C. and Hubbell, J.H. (1992) X-ray absorption (or attenuation) coefficients. Sec. 4.2.4 in International Tables for Crystallography Vol. C, A.J.C. Wilson, Ed. (Dordrecht: Kluwer Academic Publishers) 189-206.

Cromer, D.T. and Mann, J.B. (1967a) Compton scattering factors for spherically symmetric free atoms. Los Alamos Scientific Laboratory Report LA-3689, $116 \mathrm{pp}$.

Cromer, D.T. and Mann, J.B. (1967b) Compton scattering factors for spherically symmetric free atoms. J. Chem. Phys. 47, 1892-1893.

Cromer, D.T. (1969) Compton scattering factors for aspherical free atoms. J. Chem. Phys. 50, $4857-$ 4859.

Cromer, D.T. (1971) Private communication to W.J. Veigele and J.H. Hubbell.

Cullen, D.E., Chen, M.H., Hubbell, J.H., Perkins, S.T., Plechaty, E.F., Rathkopf, J.A. and Scofield, J.H. (1989) Tables and graphs of photon-interaction cross sections from $10 \mathrm{eV}$ to $100 \mathrm{GeV}$ derived from the LLNL Evaluated Photon Data Library (EPDL), Part A: Z = 1 to 50, Part B: Z $=51$ to 100, UCRL-50400, Vol. 6, Rev. 4.

Cullen, D.E., Hubbell, J.H. and Kissel, L. (1997) EPDL97: the evaluated photon data library, '97 version. UCRL-50400, Vol. 6, Rev. 5.

Deb, A. and Chatterjee, A.K. (1996a) Compton profile of vanadium carbide and vanadium nitride. Phys. Rev. B 53, 13393-13399.

Deb, A. and Chatterjee, A.K. (1996b) Compton profile of cuprous oxide by linear combination of Gaussian orbitals. J. Phys.: Condens. Matter 8, 5139-5148.

Deb, A. (1997) Experimental and theoretical investigation of Compton profile of transition metal compounds. Ph.D. Thesis, Jadavpur Univ., Calcutta.

Deb, A., Saha, S.K., Guin, H. and Chatterjee, A.K. (1999) Compton scattering studies of the electron momentum distribution in indium phosphide. Rad. Phys. Chem. (in press).

Debye, P. (1923) Zerstreuung von Röntgenstrahlen und Quantentheorie. Physik. Z. 24, 161-166.

Di Lazzaro, M.A. and Missoni, G. (1966) The spectral distribution of photons scattered by electrons bound in the K shell. Rapp. Lab. Fis. Ist. Sup. Sanita (Roma), ISS 66/6, 66/7, 66/8.

Dirac, P.A.M. (1926) Relativity quantum mechanics with an application to Compton scattering. Proc. Roy. Soc. London A 111, 405-423.

Donovan, B. and March, N.H. (1956) Momentum distribution of electrons in solids. II: Some general results with an application to metallic lithium. Proc. Phys. Soc. A 69, 1249-1260. 
DuMond, J.W.M. (1929) Compton modified line structure and its relation to the electron theory of solid bodies. Phys. Rev. 33, 643-58.

DuMond, J.W.M. (1930) Breadth of the Compton modified line. Phys. Rev. 36, 146-147.

DuMond, J.W.M. and Kirkpartick. H.A. (1931) Experimental evidence for electron velocities as the cause of Compton line breadth with the multicrystal spectrograph. Phys. Rev. 37, 136-159.

DuMond, J.W.M. (1933) The linear momenta of electrons in atoms and in solid bodies as revealed by x-ray scattering. Rev. Mod. Phys. 5, 1-33.

DuMond, J.W.M. and Kirkpatrick, H.A. (1937) A direct spectrum and shift of the Compton line with helium gas as the scatterer. Phys. Rev. 52, 419-436.

Duncanson, W.E. and Coulson, C.A. (1945) Theoretical shape of the Compton profile for atoms from $\mathrm{H}$ to Ne. Proc. Phys. Soc. 57, 190-198.

Eisenberger, P. (1970) Electron momentum density of He and $\mathrm{H}_{2}$; Compton x-ray scattering. Phys. Rev. A 2, 1678-1686.

Eisenberger, P. (1972a) Compton-profile measurements of $\mathrm{N}_{2}, \mathrm{O}_{2}$, and $\mathrm{Ne}$ using silver and molybdenum X-rays. Phys. Rev. A 5, 628-635.

Eisenberger, P., Henneker, W.H. and Cade, P.E. (1972b) Compton scattering of $x$ rays from Ne, $\mathrm{N}_{2}$, and $\mathrm{O}_{2}:$ A comparison of theory and experiment. J. Chem. Phys. 56, 1207-1209.

Eisenberger, P. and Reed, W.A. (1972c) Gamma-ray Compton scattering: Experimental Compton profiles. Phys. Rev. A 5, 2085-2093.

Eisenberger, P., Lam, L., Platzman, P.M. and Schmidt, P. (1972d) X-ray Compton profiles of Li and Na: Theory and experiments. Phys. Rev. B 6, 3671-3681.

Eisenberger, P. and Reed, W.A. (1974) Gamma-ray Compton profiles of copper and nickel. Phys. Rev. B 9, 3242-3247.

Epstein, I.R. (1973) Compton scattering and the chemistry of momentum space. Accounts of Chem. Res. 6, 145-152.

Franz, W. (1937) Über die Form der Comptonlinie. II, Ann. Physik (Leipzig) 29, 721-740.

Goldberger, M.L. and Watson, K.M. (1964) Collision Theory (Wiley, New York) p. 80.

Gray, J.A. (1920) The scattering of x- and $\gamma$-rays. J. Franklin Inst. 190, 633-655.

Halonen, V., Epstein, I.R., Tanner, A.C. and williams, B.G. (1977) Multiple scattering. Ch. 4 in B. Williams (ed.) Compton Scattering (McGraw-Hill, New York) pp. 79-101.

Heisenberg, W. (1943a) Die "beobachtbaren Grössen" in der Theorie der Elementarteilchen. Z. Physik 120, 513-538. 
Heisenberg, W. (1943b) Die beobachtbaren Grössen in der Theorie der Elementarteilchen. II. Z. Physik 120, 673-702.

Hicks, B. (1937) The shape of the Compton line for helium and molecular hydrogen. Phys. Rev. 52 , $436-442$.

Hubbell, J.H., Veigele, Wm.J., Briggs, E.A., Brown, R.T., Cromer, D.T. and Howerton, R.J. (1975) Atomic form factors, incoherent scattering functions, and photon scattering cross sections. J. Phys. Chem. Ref. Data 4, 471; erratum in (1977) 6, 615.

Hubbell, J.H., Gimm, H.A. and Øverbø, I. (1980) Pair, triplet and total atomic cross sections (and mass attenuation coefficients) for $1 \mathrm{MeV}-100 \mathrm{GeV}$ photons in elements $Z=1$ to 100 . $\mathrm{J}$. Phys. Chem. Ref. Data 9, 1023-1147.

Hubbell, J.H. and Seltzer, S.M. (1995) Tables of X-ray mass attenuation coefficients and mass energy-absorption coefficients $1 \mathrm{keV}$ to $20 \mathrm{MeV}$ for elements $Z=1$ to 92 and 48 additional substances of dosimetric interest. National Institute of Standards and Technology report NISTIR 5632.

Hubbell, J.H. (1997) Summary of existing information on the incoherent scattering of photons, particularly on the validity of the use of the incoherent scattering function. Radiat. Phys. Chem. 50, 113-124.

Jauncey, G.E.M. (1925a) Quantum theory of the unmodified spectrum line in the Compton effect. Phys. Rev. 25, 314-321.

Jauncey, G.E.M. (1925b) Quantum theory of the intensity of the modified band in the Compton effect. Phys. Rev. 25, 723-736.

Jauncey, G.E.M. (1934) Note on the structure of the Compton modified band. Phys. Rev. 46, 667668.

Kahane, S. (1998) Relativistic Dirac-Hartree-Fock photon incoherent scattering functions. At. Data Nucl. Data Tables 68, 323-347.

Kane, P.P., Basavaraju, G., Lad, S.M., Varier, K.M., Kissel, L. and Pratt, R.H. (1987) Inelastic and anomalous elastic scattering of 88.03-keV $\gamma$ rays. Phys. Rev. A 36, 5626-5631.

Kane, P.P. (1992) Inelastic scattering of X-rays and gamma rays by inner shell electrons. Physics Reports 218, 67-139.

Kane, P.P. (1997) Experimental studies of inelastic $x$-ray and $\gamma$-ray scattering. Radiat. Phys. Chem. $50,31-62$.

Kappeler, H. (1936) Messung der Form und Breite der Comptonlinie für die Gase $\mathrm{Ne}, \mathrm{O}_{2}, \mathrm{~N}_{2}$ und für die festen Stoffe Li, C und NaF. Ann. Physik 27, 129-167.

Kilby, G.E. (1961) Coherent and incoherent scattering of $\mathrm{x}$-rays by $\mathrm{H}_{2}$ and He. Proc. Phys. Soc. 78, 673-680. 
Kilby, G.E. (1963) Momentum distribution of electrons in metallic lithium. Proc. Phys. Soc. 82, 900902.

Kilby, G.E. (1965) A wave-mechanical derivation of the intensity distribution of the Compton line. Proc. Phys. Soc. 86, 1037-1040.

Kirkpatrick, H.A. and DuMond, J.W.M. (1938) Shape of the modified Compton line for hydrogen and Ceylon graphite scatterers. Phys. Rev. 54, 802-808.

Klein, O. and Nishina, Y. (1929) Über die Streuung von Strahlung durch freie Elektronen nach der neuen relativistischen Quantendynamik von Dirac. Z. Physik 52, 853-868.

Laval, J. (1942) La diffusion des rayons X par effet Compton est moindre pour un atome engagé dans un cristal que pour le même atome libre. Compt. Rend. 215, 278-279.

Manninen, S. Paakkari, T. and Kajantie, K. (1974) Gamma ray Compton profile of aluminium. Phil. Mag. 29, 167-178.

Manninen, S. and Paakkari, T. (1978) A high intensity apparatus for accurate Compton profile measurements. Nucl. Instr. Meth. 155, 115-119.

March, N.H. (1954) Momentum distribution of electrons in solids: Results for some metals using the Thomas-Fermi method. Proc. Phys. Soc. A 67, 9-16.

Mendelsohn, L. and Smith, V.H. (1977) Atoms. Ch. 5 in B. Williams (ed.) Compton Scattering (McGraw-Hill, New York) pp. 102-138.

Michels, W.C. (1961) Editor, The International Dictioary of Physics and Electronics (Van Nostrom, Princeton) p. 725.

Motz, J.W. and Missoni, G. (1961) Compton scattering by K-shell electrons. Phys. Rev. 124, 14581468.

Namito, Y., Ban, S. and Hirayama, H. (1994) Implementation of the Doppler broadening of a Compton-scattered photon into the EGS4 code. Nucl. Instr. Meth. A 349, 489-494.

Namito, Y., Ban, S., Hirayama, H., Nariyama, N., Nakashima, H., Nakane, Y., Sakamoto, Y., Sasamoto, N., Asano, Y. and Tanaka, S. (1995) Compton scattering of 20- to 40-keV photons. Phys. Rev. A 51, 3036-3043.

Nelson, W.R., Hirayama, H. and Rogers, D.W.O. (1985) The EGS4 code system. Stanford Linear Accelerator Center Report SLAC-265.

Newton, R.G. (1982) Scattering Theory of Waves and Particles 2nd ed. (Springer, New York) p. 52.

Ohmura, Y. and Matsudaira, N. (1964) Influence of Coulomb correlations on the scattering of X-ray by an electron gas. I. RPA approximation, J.PPys. Soc. Japan 19, 1355-1360. 
Owen, D.A. (1977) Compton scattering from electrons in bound states: Binding and "off-mass"-shell effects. Phys. Rev. A 16, 1594-1611.

Owen, D.A. (1978) The Compton profile dependence on "off-mass-shell" and binding effects for bound electrons. Phys. Lett. A 69, 177-179.

Paakkari, T., Manninen, S., Inkinen, O. and Liukkonen, E. (1972) Electron momentum distribution in vanadium. Phys. Rev. B 6, 351-356.

Paakkari, T., Suortti. P., Halonen, V. and Manninen, S. (1973a) Anisotropy of the electron momentum distribution in vanadium. Phys. Fenn. 8, 93-98.

Paakkari, T. and Manninen, S. (1973b) Experimental Compton profile of iron. Phys. Rev. B 8, $3765-$ 3768.

Paatero, P., Manninen, S. and Paakkari, T. (1974) Deconvolution in Compton profile measurements. Phil. Mag. 30, 1281-1294.

Pattison, P. and Schneider, J.R. (1979) Test of the relativistic 1s wavefunctions in $\mathrm{Au}$ and $\mathrm{Pb}$ using experimental Compton profiles. J. Phys. B 12, 4013-4019.

Phillips, W.C. and Weiss, R.J. (1966) X-ray determination of electron momenta in Li, Be, B, Na, $\mathrm{Mg}, \mathrm{Al}$, and LiF. Phys. Rev. 171, 790-800.

Phillips, W.C and Weiss, R.J. (1972a) Compton profile of polycrystalline lithium. Phys. Rev. B 5, 755-757.

Phillips, W.C. and Weiss, R.J. (1972b) Compton profile and electron distribution in iron. Phys. Rev. B 6, 4213-4219.

Phillips, W.C. (1973) Compton profile of single-crystal vanadium. Phys. Rev. B 7, 1047-1052.

Platzman, P. and Tzoar, N. (1977) Theory. Ch. 2 in B. Williams (ed.) Compton Scattering (McGraw-Hill, New York) pp. 28-42.

Pratt, R.H., Kissel, L. and Bergstrom, P.M. (1994) New relativistic S-matrix results for scattering beyond the usual anomalous factors/beyond impulse approximation. In G. Materlik, C.J. Sparks and K. Fischer (eds.) Resonant Anomalous X-Ray Scattering. Theory and Applications (Elsevier) pp. 9-33.

Reed, W.A. and Eisenberger, P. (1972) Gamma-ray Compton profiles of diamond, silicon, and germanium. Phys. Rev. B 6, 4596-4604.

Reineking, A., Wenskus, R., Baumann, A., Schaupp, D., Rullhusen, P., Smend, F. and Schumacher, M. (1983) Compton scattering: test of the double differential cross section in the relativistic impulse approximation. Phys. Lett. A 95, 29-31.

Ribberfors, R. (1975a) Relationship of the relativistic Compton cross section to the momentum distribution of bound electron states. Phys. Rev. A 12, 2067-2074. 
Ribberfors, R. (1975b) Relationship of the relativistic Compton cross section to the momentum distribution of bound electron states. II. Effects of anisotropy and polarization. Phys. Rev. A $12,3136-3141$.

Ribberfors, R. and Berggren, K.-F. (1982) Incoherent-x-ray-scattering functions and cross sections $\left(d \sigma / d \Omega^{\prime}\right)_{\text {incoh }}$ by means of a pocket calculator. Phys. Rev. A 26, 3325-3333.

Ribberfors, R. (1999) Personal communication.

Rodberg, L.S. and Thaler, R.M. (1967) Ch. 9. The S Matrix and the K Matrix, in Introduction to the Quantum Theory of Scattering (Academic Press, New York) pp. 227-248.

Rullhusen, P. and Schumacher, M. (1976) Cross section profiles for Compton scattering of 279.2 $\mathrm{keV}$ photons by copper, tin and lead. J. Phys. B 9, 2435-2446.

Sakurai, Y., Tanaka, Y., Bansil, A., Kaprzyk, S., Stewart, A.T., Nagashima, Y., Hyodo, T., Nanao, S., Kawata, H. and Shiotani, N. (1995) High-resolution Compton scattering study of Li: Asphericity of the Fermi surface and electron correlation effects. Phys. Rev. Lett. 74, 2252-2255.

Schülke, W., Berg, U. and Brümmer, O. (1969) Evidence for the Coulomb interaction of Be valence electrons by Compton scattering cross-section measurements. Phys. Stat. Sol. 35, 227-236.

Schülke, W., Stutz, G., Wohlert, F. and Kaprolat, A. (1996) Electron momentum-space densities of Li metal: A high-resolution Compton-scattering study. Phys. Rev. B 54, 14381-14395.

Schumacher, M. (1971) Compton scattering of gamma rays by electrons bound in heavy atoms. Z Physik 242, 444-457.

Schnaidt, F. (1934) Über das kontinuierliche und diskontinuierliche Comptonspectrum bei Wasserstoff. Ann. Physik (Leipzig) 21, 89-112.

Seltzer, S.M. (1993) Calculation of photon mass energy-transfer and mass energy-absorption coefficients. Rad. Res. 136, 147-170.

Stuewer, R.H. and Cooper, M.J. (1977) History. Ch. 1 in B. Williams (ed.) Compton Scattering (McGraw-Hill, New York) pp. 1-27.

Surić, T., Bergstrom, P.M., Pisk, K. and Pratt, R.H. (1991) Compton scattering of photons by innershell electrons. Phys. Rev. Lett. 67, 189-192.

Surić, T. (1992) Compton scattering from bound electrons: comparisons of the impulse approximation with exact IPA calculation. Nucl. Instr. Meth. A 314, 240-243.

Surić, T., Pisk, K. and Pratt, R.H. (1996) Charge dependence of the ratio of double to total ionization of a helium-like ion by Compton scattering of a high energy photon. Phys. Lett. A 211, 289-296. 
Tanner, A.C. and Epstein, I.R. (1974) Compton profile and electron momentum distribution of water. J. Chem. Phys. 61, 4251-4257.

Varma, J. and Eswaran, M.A. (1962) Inelastic scattering of gamma rays by bound electrons. Phys. Rev. 127, 1197-1203.

Veigele, Wm.J., Tracy, P.T. and Henry, E.M. (1966) Compton effect and electron binding, Am. J. Phys. 34, 1116-1121.

Veigele, Wm.J., Tracy, P.T. and Henry, E.M. (1969) Generalized Compton equation. Am. J. Phys. 37, 806-808.

Waller, I. (1927) On the scattering of radiation from atoms. Phil. Mag. 4, 1228-1237.

Waller, I. and Hartree, D.R. (1929) On the intensity of total scattering of x-rays. Proc. Roy. Soc. London A 124, 119-142.

Weiss, R.J., Harvey, A. and Phillips, W.C. (1968a) Compton line shapes from Hartree-Fock wave functions. Phil. Mag. 17, 241-253.

Weiss, R.J. and Phillips, W.C. (1968b) X-ray determination of the electron momentum density in diamond, graphite, and carbon black. Phys. Rev. 176, 900-904.

Weiss, R.J. (1972) Electron momentum distribution in silicon. Phil. Mag. 26, 153-159.

Weiss, R.J. (1973a) The Compton profiles of $\mathrm{NaF}, \mathrm{KCl}, \mathrm{S}, \mathrm{Si}_{3} \mathrm{~N}_{4}$, Ca and Co. Phil. Mag. 28, 993995.

Weiss, R.J. (1973b) Normalization of Compton profiles. Phil. Mag. 28, 1161-1163.

Weiss, R.J. (1975) The Compton profile and Compton defect in aluminium. Phil. Mag. 32, 247-249.

Weiss, R.J., Reed, W.A. and Pattison, P. (1977) Experimentation. Ch. 3 in B. Williams (ed.) Compton Scattering (McGraw-Hill, New York) pp.43-78.

Wenskus, R., Baumann, A., Rullhusen, P., Schaupp, D., Smend, F. and Schumacher, M. (1985) Relativistic double differential cross sections for Compton scattering by bound electrons: Test at low energies and predictions at intermediate photon energies. Z. Phys. A 320, 179-184.

Wentzel, G. (1927a) Zur Theorie des Comptoneffekts. I. Z. Physik 43, 1-8.

Wentzel, G. (1927b) Zur Theorie des Comptoneffekts. II. Z. Physik 43, 779-787.

Weyrich, W., Pattison, P. and Williams, B.G. (1979) Fourier analysis of the Compton profile: Atoms and molecules. Chem. Phys. 41, 271-284.

Wheeler, J.A. (1937) On the mathematical description of light nuclei by the method of resonating group structure. Phys. Rev. 52, 1107-1122. 
Whittingham, I.B. (1971) Incoherent scattering of gamma rays in heavy atoms. J. Phys. A 4, 21-37.

Whittingham, I.B. (1981) Compton scattering of 279.1 and $661.6 \mathrm{keV}$ photons. Austral. J. Phys. 34, $163-183$.

Williams, B.G. (Project Coordinator), Felsteiner, J., Halonen, V., Paakkari, T., Manninen, S., Reed, W., Eisenberger, P., Weiss, R.J., Pattison, P. and Cooper, M.J. (1976) International Union of Crystallography Commission on Spin, Charge and Momentum Density. Project No. 1. Standardization of Compton profile measurements - The Compton profile of water. Acta Cryst. A 32, 513-526.

Williams, B.G. (1977a) Compton Scattering (McGraw-Hill, New York).

Williams, B.G. (1977b) The experimental determination of electron momentum densities. Phys. Scripta 15, 92-111.

Wittwer, L.A. (1972) K-shell Compton scattering on tin and gold at $145 \mathrm{keV}$ and gold at $320 \mathrm{keV}$. Lawrence Livermore Laboratory Report UCRL-51268. 

